\title{
Title: Correlation of Flow Cytometry and Laser Scanning Confocal Microscopy Data: PMTs, CVs, Dichroics, Optics and Spectral Data.
}

\section{Robert M. Zucker PhD}

Reproductive Toxicology Division, National Health and Environmental Effects Research Laboratory, U.S. Environmental Protection Agency, Research Triangle Park, North Carolina 27711

Flow cytometry (FCM) and laser scanning confocal microscopy (CLSM) are two technologies, which are interrelated as they use many of the same components (1-3). Both technologies use a combination of lasers, filters/dichroics to excite a sample and PMTs to detect the fluorescent emission. In each technology, a sample is stained with a fluorescent dye and excited with a specific wavelength of laser light. The emitted fluorescence travels through optical elements, and is detected with PMTs. The data is presented as intensity values with both technologies. Data learned from one technology could influence the operation of the other technology. Measurements are made on cell structure in CLSM and are made on individual cells with FCM. In both technologies cell structure data can be obtained by using non-fluorescent techniques of light scatter (FCM) or interference contrast (CLSM).

Different examples of FCM and CLSM correlation will be described.

Example \#1: A cell can be characterized by its fluorescent emission and sorted by FACS. The sorted cell can then be analyzed by CLSM for its structure and function.

Example \#2: The FCM measures the cell as a unit. It quantifies the number and intensity of cells that contain a specific probe. The CLSM can define the probe's spatial distribution within a cell. Both technologies can use similar staining logic, similar filters/ dichroics, and the same excitation/emission wavelengths.

Example \#3 The coefficient of variation (CV) is defined as the standard deviation $(\sigma)$ of the fluorescent intensity of a population of beads or pixels expressed as a proportion or percentage of the mean $(\mu)$ intensity $(\mathrm{CV}=\sigma / \mu)$. FCM has historically used the $\mathrm{CV}$ concept to align the flow cytometer and insure the flow cell is clean with normally flowing fluidics. In this $\mathrm{CV}$ assay, we suspend beads in solution and place the bead peak around channel 400. The CV's are then measured at low and high flow rates. A low CV with a symmetrical histogram should be obtained with both flow rates. Usually a fluidic problem will distort the histogram and widen the CV.

In CLSM, 100,000 pixels were measured from within one homogeneous Spherotech 10 micron bead or substrate fluorescent slide. This is analogous to measuring 10,000 intensity beads with FCM. The CV of pixel intensities is dependent on a number of machine variables that include frame averaging, photo multiplier tube (PMT) voltage, and laser stability and laser power. The lower the $\mathrm{CV}$ of an image, the less averaging that will be necessary for obtaining a good image. If a system is aligned properly it will use photons effectively allowing the PMTs to be operated with lower voltages thus yielding a better image with less averaging. The $\mathrm{CV}$ method can help detect alignment problems in both a CLSM and FCM technologies. The CV measurement 
can also be used to evaluate the quality of the PMTs in both FCM and CLSM (1-3) and the stability of different laser lines as shown in Figure 1.

Example 4: Spectral Scanning. A Leica spectral scan of 50 increments ( $5 \mathrm{~nm}$ each) between 400 and 650nm was made with a 10x Plan Apo lens and a LightForm reference light source positioned on the stage. This lamp contains specific peaks similar to a mercury arc emission lamp. The PMT revealing the tightest peaks (low $\mathrm{CV}$ ) and the largest valleys between peaks yielded the best spectral response. This test appears to measure the PMT sensitivity and alignment in the different spectral ranges and is consistent with the CV principles discussed previously for FCM and CLSM.

Other factors relevant to CLSM are field illumination spectral registration, axial resolution, laser noise (short term and long term), laser stability, system sensitivity, and PMT functionality (1-3). These factors have been evaluated on CLSM with equivalent counterparts sometimes existing in FCM.

\section{References}

1. Zucker RM and Price OT: Methods 18: 447-458 1999

2. Zucker RM and Price OT: Cytometry 44:273-294 2001

3. Zucker RM and Price OT: Cytometry 44: 295-308 2001

This abstract of a proposed presentation does not necessarily reflect EPA policy

Short Term Laser and Transmission PMT Variation (T< 1 Second) ( $P M T=200 \mathrm{~V})$

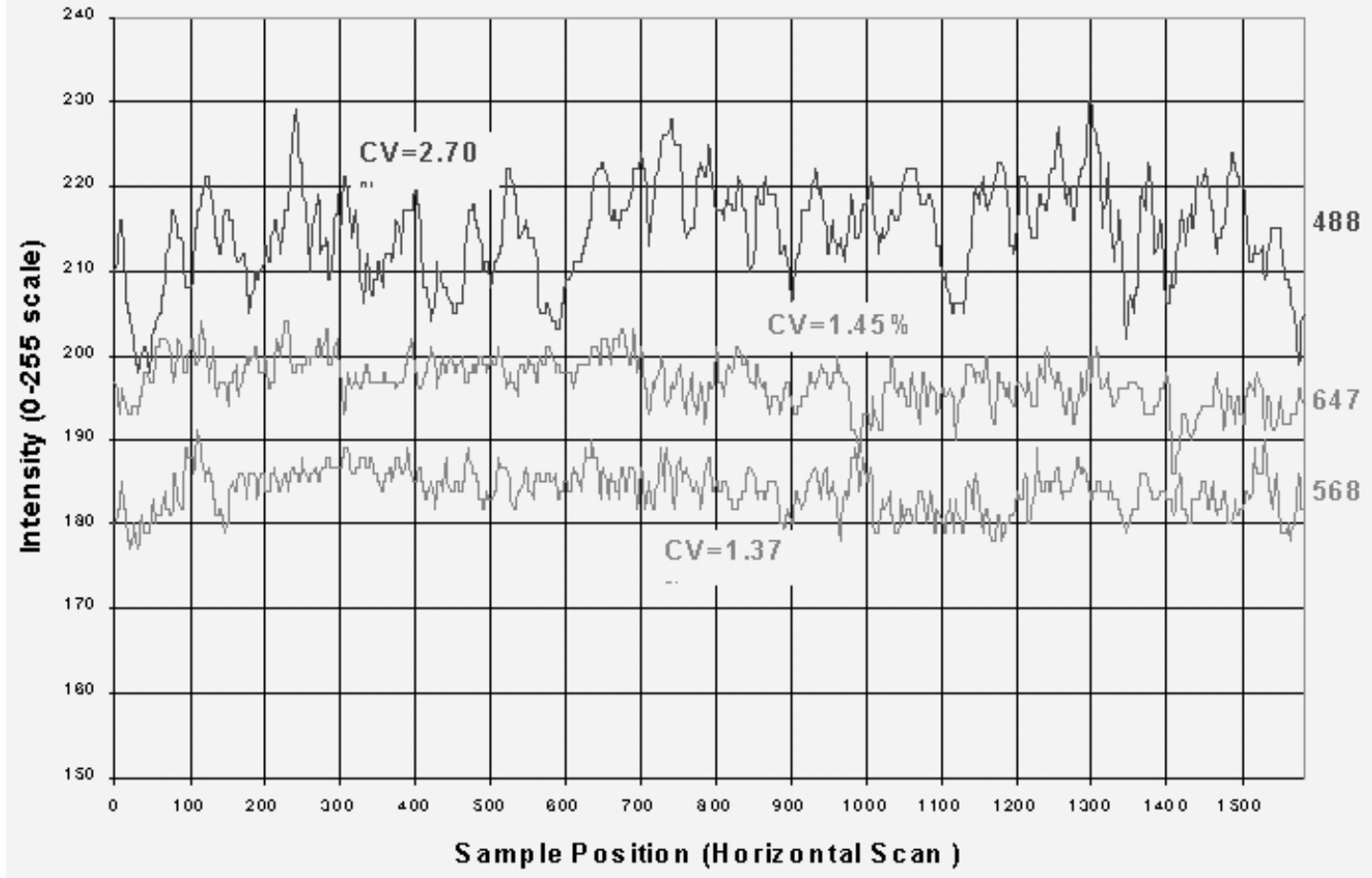

Fig 1. The Argon Krypton laser in a Leica SP1 CLSM was used. To obtain an image, the transmission PMT was kept constant and laser intensity was varied with the AOTF. A single line scan was measured from the images (shown above). The intensity variations of the three wavelength line scans were different. The 488 line $(\mathrm{CV}=2.70)$ is almost twice as noisy as the $568(\mathrm{CV}=1.45)$ and $647(\mathrm{CV}=1.37)$ lines. 